\title{
Non-Halal biomarkers identification based on Fourier Transform Infrared Spectroscopy (FTIR) and Gas Chromatography-Time of Flight Mass Spectroscopy (GC-TOF MS) techniques
}

\author{
Gunawan Witjaksono ${ }^{1, *}$, Irwan Saputra ${ }^{2}$, Marsad Latief $^{3}$, Irwandi Jaswir ${ }^{2}$, Rini Akmeliawati ${ }^{3}$, and Almur Abdelkreem \\ Saeed Rabih ${ }^{1}$ \\ ${ }^{1}$ Department of Electrical and Electronic Engineering, Universiti Teknologi PETRONAS, Seri Iskandar, 32610, Perak Darul Ridzuan, \\ Malaysia \\ ${ }^{2}$ International Institute for Halal Research \& Training (INHART), International Islamic University Malaysia (IIUM) \\ ${ }^{3}$ Department of Mechatronics Engineering, International Islamic University Malaysia (IIUM) \\ P.O Box 10, 50728 Kuala Lumpur, Malaysia
}

\begin{abstract}
Consumption of meat from halal (lawful) sources is essential for Muslims. The identification of non-halal meat is one of the main issues that face consumers in meat markets, especially in non-Islamic countries. Pig is one of the non-halal sources of meat, and hence pig meat and its derivatives are forbidden for Muslims to consume. Although several studies have been conducted to identify the biomarkers for nonhalal meats like pig meat, these studies are still in their infancy stages, and as a result there is no universal biomarker which could be used for clear cut identification. The purpose of this paper is to use Fourier Transform Infrared Spectroscopy (FTIR) and Gas Chromatography- Time of Flight Mass Spectroscopy (GC-TOF MS) techniques to study fat of pig, cow, lamb and chicken to find possible biomarkers for pig fat (lard) identification. FTIR results showed that lard and chicken fat have unique peaks at wavenumbers $1159.6 \mathrm{~cm}^{-1}, 1743.4 \mathrm{~cm}^{-1}, 2853.1 \mathrm{~cm}^{-1}$ and $2922.5 \mathrm{~cm}^{-1}$ compared to lamb and beef fats which did not show peaks at these wavenumbers. On the other hand, GC/MS-TOF results showed that the concentration of 1,2,3-trimethyl- Benzene, Indane, and Undecane in lard are 250, 14.5 and 1.28 times higher than their concentrations in chicken fat, respectively, and 91.4, 2.3 and 1.24 times higher than their concentrations in cow fat, respectively. These initial results clearly indicate that there is a possibility to find biomarkers for non-halal identification.
\end{abstract}

\section{Introduction}

Authenticity of food has become a paramount concern for both food industries and consumers [1]. The food industries have legal compliance toward selling the right food for the right price, maintaining well-defined quality and using safe ingredients [2]. On the other hands, for consumers transparency in food specification is an ever increasing demand and essential due to religion credence, hard-earned fortunes [3] and some people's allergy to some types of foods [4]. For raw meat markets, the common practice is that consumers rely on their visual inspection to differentiate between meat species. However, in most cases meat types differ subtly in the visual appearance, which make it more difficult to differentiate [5]. Furthermore, in case of mixed and processed meat the differentiation based on appearance will be quite impossible [6]. Regulatory agencies as a third party also concern about the food authenticity due to that food adulteration could bring revenue losses to the state. Research scientists have been conducting continuous studies to support the regulatory and commercial initiatives to develop analytical techniques

\footnotetext{
*Corresponding author: gunawan.witjaksono@utp.edu.my
}

to detect the fraudulent foods and the ingredients that breach the labelled marks [7]. From the religious perspective, most religions in the world urge their followers to comply with certain dietary laws. For instance, Islam encourages Muslims to consume only Halal (lawful) foods, which exclude some types of food such as pig meat and its derivatives like lard, ham, bacon, pork and sausage, except in very extreme conditions. Halal food is expected to reach $20 \%$ of the world trade as the Muslims are expected to make $30 \%$ of the world populations by the year 2025 [8]. Judaism has a defined dietary law (kashrut) for the Jewish to follow, which also prohibits pig meat and its derivatives [9], and in Hindu religion consumption of beef and its products is not allowed [10]. In many countries, food manufacturers use lard to adulterate oil ingredients due to the cheap price of lard [11]. In 2013, there was a big scandal that hit Europe, where beef had been replaced by horsemeat, and the later had been sold with beef labelling. In addition to Europe, Asia also witnessed scandals in Malaysia where chocolate and pharmaceutical preparations were found to contain pork traces in 2013, and 2014, respectively [12]. In some other Asian 
countries like India it is common to find buffalo meat mixed with other meat species due to its lower cost and availability, which will be very hard to identify especially if it is mixed with beef or if it is the processed form [13]. Beside meat markets, some manufacturers of dairy adulterate butter with some cheap fats like chicken fats, lard and mutton fats to gain more profits [14]. Due to aforementioned issues, food authentication has become a paramount concern for everyone. Various methods have been developed for food species identification. Some of these methods include protein based methods like electrophoretic techniques, chromatographic, immune assays and DNA as a molecular detection method [15]. In addition, electronic nose, polymerase chain reaction and spectroscopic techniques such as ultraviolet (UV), near infrared (NIR), mid infrared (MIR), visible light, and Raman spectroscopy are widely used [16]. IR based techniques like Fourier Transform Infrared Spectroscopy (FTIR) is reliable for meat sample identification due to its capability of using as fingerprint technique when it is combined with powerful chemometric techniques [17]. This paper reports a study on Pig fat (lard), chicken fat, cow fat and lamb fat using FTIR and Gas Chromatography-Time of Flight Mass Spectroscopy (GC-TOFMS) to find non-halal biomarkers for halal identification.

\section{Methods and Samples Preparation}

Two methods were used to study the non-halal biomarkers. Firstly FTIR was used, and then the results were confirmed by using GC-TOFMS.

\subsection{Fourier Transform Infrared Spectroscopy (FTIR)}

Infrared (IR) spectroscopy is a rapid, non-destructive, sensitive and easy sample preparation technique that is being used for many years in food sector for analysis and classification of foods. It is used either in near infrared (NIR) or in mid infrared bands (MIR) [1]. The advantage of using Infrared (IR) techniques for meat samples identification is that they provide much information, with a unique absorption bands for the functional groups that unique to the meat sample studied [3]. Fourier Transform Infrared Spectroscopy (FTIR) is a modern technique derived from the conventional IR spectroscopy, and it works in the MIR region $\left(400-4000 \mathrm{~cm}^{-1}\right)$. It has a powerful capability for food analysis and investigating its individual components due to the high energy throughput, excellent wavenumber reproducibility and accuracy [18].

\subsubsection{FTIR meat samples preparation and fats extraction}

Total sixteen meat samples from pig, chicken, lamb and cow were collected from a local slaughterhouse at Gombak Market in Malaysia. Each meat type contains five samples. The preparation started firstly by washing the samples by distilled water to remove any contamination that might stuck on the surface of the meat samples. Then, the meat samples were cut into small element $(1 \mathrm{~cm} \times 1 \mathrm{~cm})$ and kept at $-20^{\circ} \mathrm{C}$ until they are used for the fat extraction process to obtain fats of the different samples. Pig fat (Lard) and chicken fat, lamb fat, and cow fat were extracted by rendering the adipose tissues according to the method described by Che Man et al [19]. All chemicals used in this experiment were of analytical grade. The pure extracted fats were then analyzed by means of FTIR spectroscopy.

\subsubsection{GC-TOF MS meat samples preparation and fats extraction}

For this study total nine meat samples of pig, chicken and cow meats were collected, by having three samples for each meat type. Preparation of the meat samples and also the extraction of the fats have been conducted using the same method mentioned above for the FTIR measurements. After extracting the pure fats, each fat sample $(50 \mathrm{mg}$ ) were dissolved in $0.8 \mathrm{~mL}$ hexane. The mixture was then mixed for 1 min using a vortex mixer, and then stored in the dark at $-18{ }^{\circ} \mathrm{C}$ prior to GC-TOF MS analysis.

\section{Results and discussion}

\subsection{FTIR Results}

Nicolet iS50 FTIR Spectrometer was used to acquire the full spectrum in the mid infrared region $(400-4000$ $\left.\mathrm{cm}^{-1}\right)$. The number of scans was fixed to 32 with a resolution of $4 \mathrm{~cm}^{-1}$. The measurement was calibrated against the background air. The whole FTIR spectrum which corresponds to the stretching of the functional groups that present in the fat of the species [17] studied is shown in Fig.1. The graph shows the average spectrum of three samples for each of lard, chicken fat, cow fat and lab fat.

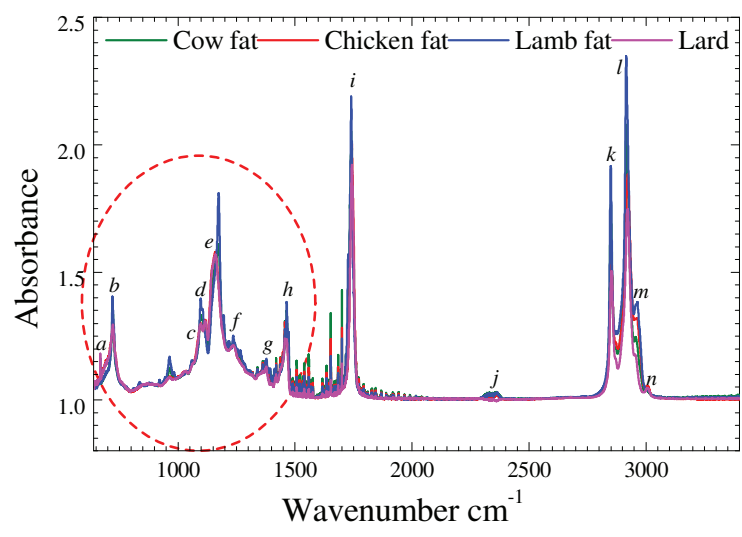

Fig. 1. FTIR Spectra of lard, chicken fat, cow fat and $1 \mathrm{mb}$ fat

From the spectra it was found that lard is different from the other fats in some peaks. The spectra shown in Fig.1 could be divided into two regions: the higher 
wavenumbers of more than $1500 \mathrm{~cm}^{-1}$ and the finger print region which is the region below $1500 \mathrm{~cm}^{-1}$.

\subsubsection{FTIR peaks in Region one: $>1500 \mathrm{~cm}^{-1}$}

At the wavenumbers higher than $1500 \mathrm{~cm}^{-1}$, lard is different from the other fats in term of the absorbance at the peaks $n, m$ and $j$ as shown in Fig. 1. Fig. 2 shows the closer look at the points $n$ and $m$. in the point $\mathrm{m}$ (shown by the red-dotted circle in Fig. 2) lard has shown a small shoulder, while chicken has a broader shoulder.

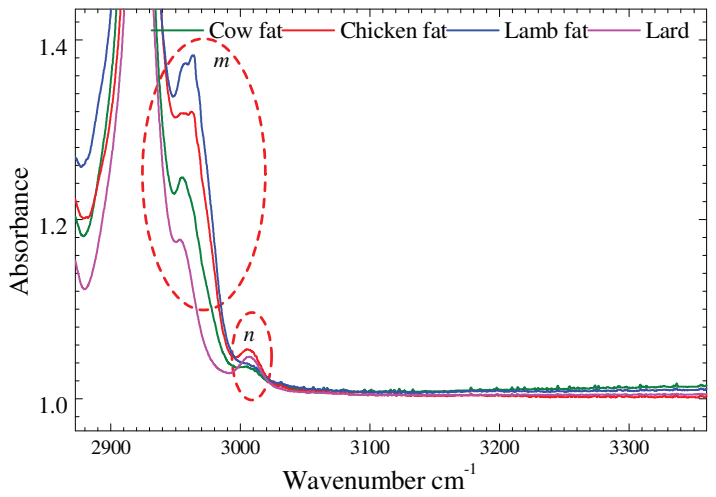

Fig. 2. FTIR Spectra of lard, chicken fat, cow fat and lamb fat at $m$ and $n$ regions

On the other hands, cow fat exhibited single peak and lamb fat has demonstrated double peaks. At the region referred by $n$ only lard and chicken fats have shown peaks, while cow and lamb fats have no peaks. Lard is again different from the other fats in the region marked by $j$ in Fig. 1. At his region lard has shown an inverted peak as explained further in Fig. 3.

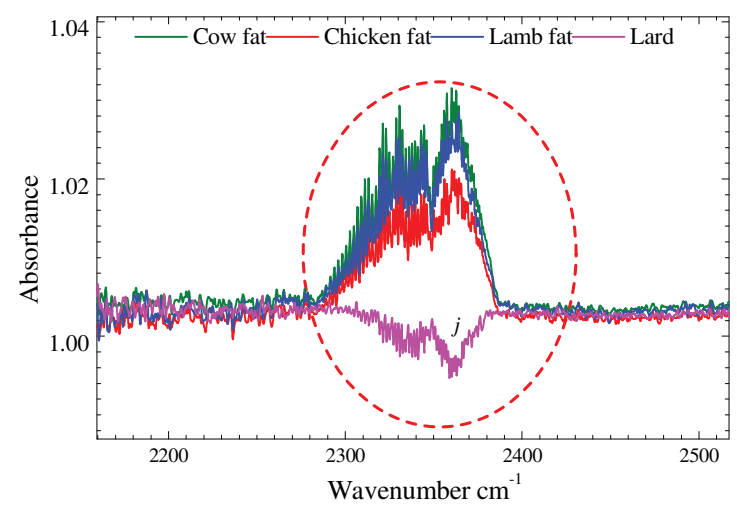

Fig. 3. An inverted peak for lard at wavenumber $2361.05 \mathrm{~cm}^{-1}$

\subsubsection{FTIR peaks in Region two: $<1500 \mathrm{~cm}^{-1}$}

For the wavenumbers less than $1500 \mathrm{~cm}^{-1}$, lard is different from the other fats at the peak $a, c, d, e$, and $h$ as shown in Figure 4. However chicken also exhibited same peaks except at the peak denoted by $a$, which is unique for lard. Hence at the fingerprint region $a$ could be the most suitable peak number to be used for lard identification.

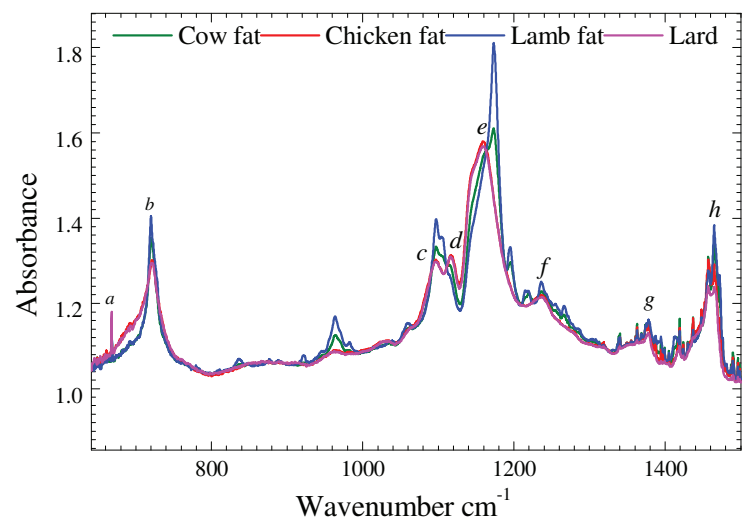

Fig. 4. Fingerprint region (wavenumber $<1500 \mathrm{~cm}^{-1}$ ) for lard identification

Fig. 5 shows representing the absorbance of the lard, cow, lamb and chicken fats using different reference levels. For clarity of differentiation in the graph only cow fat has the same original reference level, while chicken reference level is raised one level above the cow fat reference by adding 1 to the original absorbance values of the chicken fat, lamb fat is two levels above the cow fat reference by adding 2 to the original absorbance values of the lamb fat, and finally lard is three levels above the original reference line by adding 3 to the original absorbance values of lard.

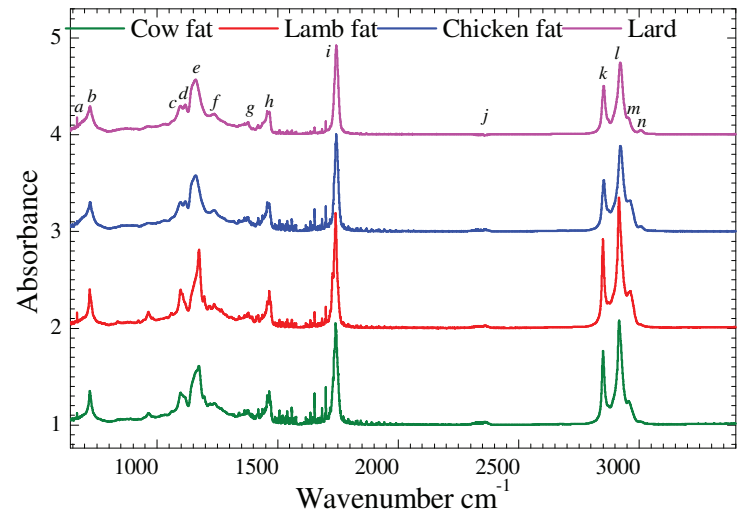

Fig. 5. FTIR Spectra of lard, chicken fat, cow fat and lamb fat

\subsection{Gas Chromatography-Time of Flight Mass Spectroscopy (GC-TOF MS) Results}

Techniques based on chromatography offer rapid and reliable sample separation for compounds analysis. GCTOF MS is known as a powerful tool for the analytical analysis of complex samples [1]. For this research Agilent 7693 B GC coupled with TOF MS was used with hp-5ms column. The study was conducted on total nine samples (three samples each) of lard, cow, lamb and chicken fats to analyze the aromatic hydrocarbons. Each fat type contains three samples. Table 1 shows the obtained average (for three samples) area covered by each hydrocarbon which represents the composition weightage for the different fat types. 
Table 1. Aromatic hydrocarbons compositions for lard, chicken and cow fats

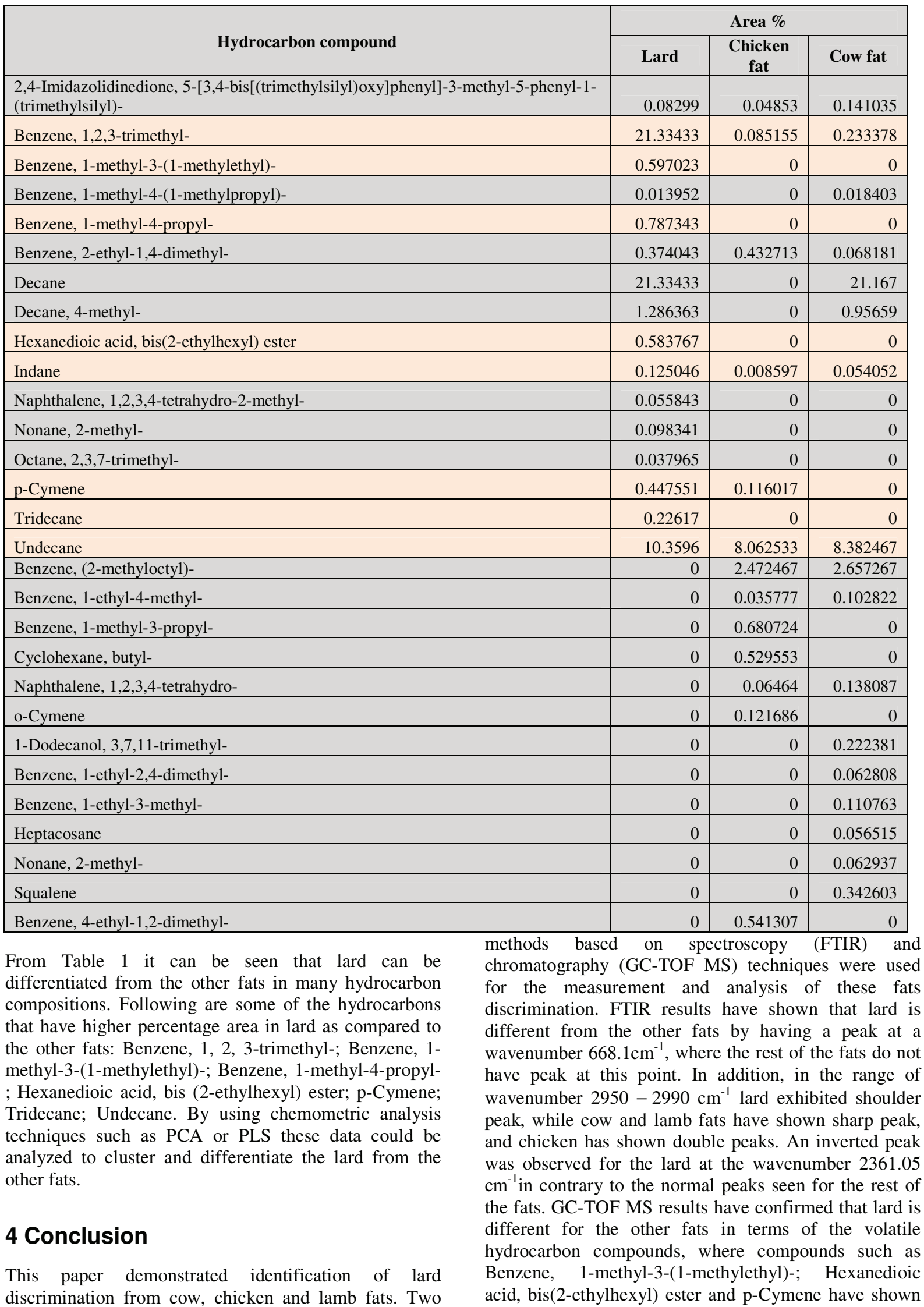


very high compositional percentage in lard compared to other fats.

The authors of this paper acknowledge the financial support by Universiti Teknologi PETRONAS (UTP) under STIRF-UTP, fund/ project code: 0153AA-F71

Ministry of Higher Education, Malaysia and International Islamic University Malaysia (IIUM) under the scheme of MyRA INCENTIVE RESEARCH GRANT SCHEME (MIRGS), Project code: MIRGS13-01-002-0002.

\section{References}

1. A. Rohman and Y. B. C. Man Food Reviews International, 18, pp. 97-112, (2013).

2. W. Kamm, F. Dionisi, C. Hischenhuber, and K.-H. Engel, Food Reviews International, 17, pp. 249-290, (2001).

3. E. Kurniawati, A. Rohman, and K. Triyana, Meat Science, 96, pp. 94-98, (2014).

4. B. Mamikoglu, Otolaryngology - Head and Neck Surgery, 133, pp. 534-537, (2005).

5. N. Z. Ballin, F. K. Vogensen, and A. H. Karlsson, Meat Science, 83, pp. 165-174, (2009).

6. S. A. Sarah, W. N. Faradalila, M. S. Salwani, I. Amin, S. A Karsani, and A. Q. Sazili, Food Chemistry, 199, pp. 157-164, (2016).
7. G. Downey, Advances in Food Authenticity Testing, 1st Edition ed.: Woodhead Publishing, (2016).

8. H. I. Abdullah Amqizal, H. A. Al-Kahtani, E. A. Ismail, K. Hayat, and I. Jaswir, Food Control, 78, pp. 297-303, (2017).

9. Schröder and M. J.A., Food Quality and Consumer Value: Delivering Food that Satisfies, (2003).

10. K. Bonne and W. Verbeke, Agriculture and Human Values, 25, pp. 35-47, (2008).

11. A. A. Aida, Y. B. Che Man, C. M. V. L. Wong, A. R. Raha, and R. Son, Meat Science, 69, pp. 47-52, (2005).

12. C. Flaudrops, N. Armstrong, D. Raoult, and E. Chabrière, Journal of Food Composition and Analysis, 41, pp. 104-112,( 2015).

13. B. G. Mane, S. K. Mendiratta, A. K. Tiwari, and K. N Bhilegaokar, Food Analytical Methods, 5, pp. 296-300,( 2012).

14. A. F. Nurrulhidayah, S. R. Arieff, A. Rohman, I. Amin, M Shuhaimi, and A. Khatib, International Food Research Journal, 22, pp. 832-839, (2015).

15. W. Xiang, Y. Shang, Q. Wang, Y. Xu, P. Zhu, K. Huang, et al., Food Chemistry, 234, pp. 472-478, (2017).

16. L. M. Reid, C. P. O'Donnell, and G. Downey, Trends in Food Science \& Technology, 17, pp. 344-353, (2006).

17. H. Rahmania, Sudjadi, and A. Rohman Meat Science, 100, pp. 301-305,(2015)

18. A. Bendini, L. Cerretani, F. D. Virgilio, P. Belloni, M. BonoliCarbognin, and G. Lercker, Journal of Food Quality, 30, pp. 424 - 437, (2007).

19. Y. B. Che Man, Z. A. Syahariza, M. E. S. Mirghani, S. Jinap, and J. Bakar, Food Chemistry, 90, pp. 815-819,( 2005). 